\title{
Stretching rates and equivalent length near the tropopause
}

\author{
R. K. Scott, ${ }^{1,2}$ E. F. Shuckburgh, ${ }^{3}$ J.-P. Cammas, ${ }^{1}$ and B. Legras ${ }^{3}$ \\ Received 27 September 2002; revised 10 February 2003; accepted 21 March 2003; published 12 July 2003.
}

[1] Diagnostics of mixing in the upper troposphere/lower stratosphere region reveal interesting seasonal and interannual variability and illustrate how the subtropical tropopause can be identified with a region of minimum mixing, a partial barrier to the transport of air between the troposphere and stratosphere. Both the strength and the location of this barrier show substantial seasonal variability, with a stronger barrier and weaker mixing observed during winter. The interannual variability of the mixing intensity near the tropopause on the $350 \mathrm{~K}$ isentropic surface suggests a correlation with the phase of the El Niño-Southern Oscillation, with weaker mixing occurring during strong El Niño years. The diagnostics are based on the analyses and reanalyses from the European Centre for Medium-Range Weather Forecasts over the 21 year period 1979-2000 and include the exponential stretching rates of material contours and Nakamura's [1996] modified Lagrangian mean effective diffusivity/equivalent length. As well as providing information about the spatial and temporal distribution of mixing intensity, the diagnostics also indicate sensitivity to changes in the analyses/reanalyses data sets. In particular, a stronger seasonal cycle and greater interannual variability is found in the more recent (1994-2000) analyses than in the earlier (1979-1993) reanalyses. INDEX TERMS: 0340 Atmospheric Composition and Structure: Middle atmosphere-composition and chemistry; 3309 Meteorology and Atmospheric Dynamics: Climatology (1620); 3362 Meteorology and Atmospheric Dynamics:

Stratosphere/troposphere interactions; KEYWORDS: upper troposhere/lower stratosphere (UTLS), tropopause, mixing, contour advection, equivalent length, interannual variability

Citation: Scott, R. K., E. F. Shuckburgh, J.-P. Cammas, and B. Legras, Stretching rates and equivalent length near the tropopause, J. Geophys. Res., 108(D13), 4394, doi:10.1029/2002JD002988, 2003.

\section{Introduction}

[2] Transport of air across the tropopause plays an important role in determining the chemical composition and radiative properties of the troposphere and stratosphere. Quantifying this transport, however, presents a significant challenge on account of the many multiscale processes involved, including the global-scale mean meridional circulation, intermediate-scale advective and convective processes, three-dimensional (3D) turbulent mixing, and molecular diffusion (see, e.g., Holton et al. [1995] for a general overview). If the tropopause can be considered as a barrier to transport, as is suggested by the strong gradients in ozone and water vapor, it is important to determine the permeability of this barrier and the general mixing properties of the tropopause region.

[3] Various barriers to transport are already known to exist in the stratosphere, in particular, the stratospheric polar

\footnotetext{
${ }^{1}$ Laboratoire d'Aérologie, Centre National de Recherche Scientifique, Toulouse, France.

${ }^{2}$ Now at Department of Applied Physics and Applied Mathematics, Columbia University, New York City, New York, USA.

${ }^{3}$ Laboratoire de Météorologie Dynamique, Ecole Normale Superieure, Paris, France.

Copyright 2003 by the American Geophysical Union. 0148-0227/03/2002JD002988
}

vortex edge [e.g., Bowman, 1993; Chen, 1994] and the subtropical edge of the stratospheric surf zone [e.g., Juckes, 1989; Polvani et al., 1995; Bowman and Hu, 1997]. Various studies have also considered the possibility of a transport barrier at the tropopause [Pierrehumbert and Yang, 1993; Chen, 1995; Appenzeller et al., 1996; Bithell and Gray, 1997]. These studies have generally involved Lagrangian diagnostics of mixing, such as particle dispersion, contour advection, and separation or stretching rates. More recent studies by Haynes and Shuckburgh [2000a, 2000b] (hereinafter referred to as HSOOa and HSOOb) and Allen and Nakamura [2001] (hereinafter referred to as AN01) have considered the structure and the strength of these barriers in terms of a modified Lagrangian mean effective diffusivity [Nakamura, 1996], a hybrid Eulerian-Lagrangian diagnostic based on an instantaneous tracer distribution. In particular, HS00b clearly identified the tropopause on middle-world isentropic surfaces with a minimum in effective diffusivity. Thus the midlatitude and subtropical tropopause appears to act as a barrier that inhibits the quasi-horizontal mixing of tropospheric and stratospheric air on isentropic surfaces by synoptic-scale eddies in the troposphere.

[4] In this paper we examine various diagnostics of mixing properties near the tropopause. The first of these, discussed in section 3, is the stretching rate of material contours, initialized by isolines of potential vorticity (PV) on isentropic surfaces and advected by winds from meteo- 
rological analyses. The methodology is similar to that used by Bithell and Gray [1997] for a 6 day period in October 1990, except that we consider stretching rates over a 21 year period from 1979-2000. The use of the longer data set enables consideration of both seasonal and interannual variability of stretching rates. It also helps explain Bithell and Gray's conclusion that stretching rates are relatively uniform regardless of the value of PV used to initialize the contour. We find that whereas this is true for the time of year considered in their study (October, i.e., Northern Hemisphere (NH) autumn), it is not true for winter months, where there is a stronger dependence of stretching rates on the $\mathrm{PV}$ value.

[5] Because the characteristics of the flow field near the tropopause differ from those near, say, the polar vortex edge, where the energy spectrum in the velocity field decays much more rapidly, Shepherd et al. [2000] have argued that Lagrangian techniques based on the chaotic advection paradigm (e.g., contour advection) may not work as well here as in the stratosphere. On the other hand, synoptic structures usually persist over several turnover times, and there is evidence from many previous works [e.g., Appenzeller et al., 1996; Baray et al., 2000] that observed mesoscale structures at or near the tropopause can, to a large extent, be understood in terms of isentropic transport. Further, because much of the stirring by baroclinic eddies can be described in a quasi-horizontal isentropic framework, the use of contour advection on isentropic surfaces provides a natural way in which to address crosstropopause transport, even when the timescales for isentropic and diabatic motion are less well separated here than in the stratosphere.

[6] The second diagnostic we consider is the equivalent length/effective diffusivity [Nakamura, 1996]. This diagnostic has been used recently to examine the annual cycle of the mixing properties near the tropopause (HS00b; AN01). We present both the equivalent length calculated, as by $\mathrm{HS} 00 \mathrm{a}$ and $\mathrm{HSOOb}$, from an advection diffusion equation for a passive tracer field (section 4) as well as the equivalent length calculated using contour advection with surgery to generate a PV distribution with fine-scale structure (section 5). This second method allows for the rapid calculation of the equivalent length on a particular day using only the PV and winds on the few days prior. The use of two separate methods also provides a useful comparison of the two diagnostics. The equivalent length is again calculated using each method over the period 1979-2000, which allows a consideration of the interannual variability of the mixing intensity near the tropopause. A discussion of interannual variability in all diagnostics and the dependence on trends in the analyses data used to calculate these is given in section 6 .

\section{Data and Numerical Methods}

\subsection{Data}

[7] Our data are taken from the European Centre for Medium-Range Weather Forecasts (ECMWF) ERA-15 reanalyses, which cover the period 1979-1993, and from the ECMWF operational analyses for the period 19942000. The reanalyses are a uniform data set in the sense that the model, model resolution, and assimilation method are uniform during the 15 year period, although there are, of course, changes in the density of the data assimilated. The model used to generate the operational analyses, on the other hand, has undergone substantial development during the period 1994-2000, which makes this set less suitable for considering interannual variability using the diagnostics presented in this paper. Changes in the analyses include the introduction of $3 \mathrm{D}$ and $4 \mathrm{D}$ variational data assimilation in 1996 and 1997, respectively [Courtier et al., 1998; Klinker et al., 2000], increases in the horizontal resolution to T213 in 1991 and T319 in 1998, and increases in the number of vertical levels to 50 and 60 in 1999, most of these concentrated in the stratosphere. The ERA-15 reanalyses [Gibson et al., 1997] were generated using a horizontal resolution of T106 with 31 levels in the vertical and a data assimilation scheme based on optimal interpolation.

[8] For the contour advection calculations we use the analyses and reanalyses with a horizontal resolution of T106, corresponding to a grid point spacing of $\sim 1.3^{\circ}$ near the equator, while for the tracer advection the horizontal resolution is T42 (see Haynes and Shuckburgh [2000a, 2000b] for a discussion of the effect of resolution in this case). The temporal resolution is 6 hourly. The vorticity, divergence, and temperature on the pressure surfaces are used to calculate the horizontal velocity and potential vorticity on the isentropic surfaces $330 \mathrm{~K}, 350 \mathrm{~K}$, and $370 \mathrm{~K}$, using a vertical interpolation based on cubic splines.

[9] For the contour advection-based diagnostics the potential vorticity on the isentropic surfaces on particular days is then used to initialize contours, which are advected with the corresponding isentropic winds using the contour advection method (see Norton [1994] and Waugh and Plumb [1994] for details). For the advection we use a fourth-order Runga-Kutta scheme, interpolating the winds in time between the 6 hourly analyses onto a 15 min time step, again using cubic splines, as described by Mariotti et al. [1997]. The importance of adequate temporal resolution of the advecting winds for the accurate generation of fine scales is discussed by Waugh and Plumb [1994].

\subsection{Stretching Rates}

[10] When the contour advection is used without surgery, that is, without the removal of features below a certain scale threshold, the stretching and folding of the material contours in regions of strong mixing leads to an exponential increase of contour length. In the tropopause region an advection time of 6 days is usually sufficient to define such an exponential stretching rate [see, e.g., Bithell and Gray, 1997]. The stretching rate is large when (and in regions where) mixing is strong and is small when mixing is weak. We obtain a picture of the time variability of the stretching rates by initializing contour advection runs three times per month (on the 2nd, 12th, and 22nd of each month) over the entire periods covered by the reanalyses and the analyses for each of the isentropic surfaces $330 \mathrm{~K}, 350 \mathrm{~K}$, and $370 \mathrm{~K}$ and in each hemisphere. In each simulation, six contours are used, initialized with potential vorticity values of $\pm 0.6,1.6$, 2.6, 3.6, 4.6, and 5.6 potential vorticity units (PVU) (where $1 \mathrm{PVU}=10^{-6} \mathrm{~m}^{2} \mathrm{~s}^{-1} \mathrm{~K} \mathrm{~kg}^{-1}$ ). Thus in addition to the time variability we also obtain information about the latitudinal location (in terms of a PV coordinate) of regions of strong 

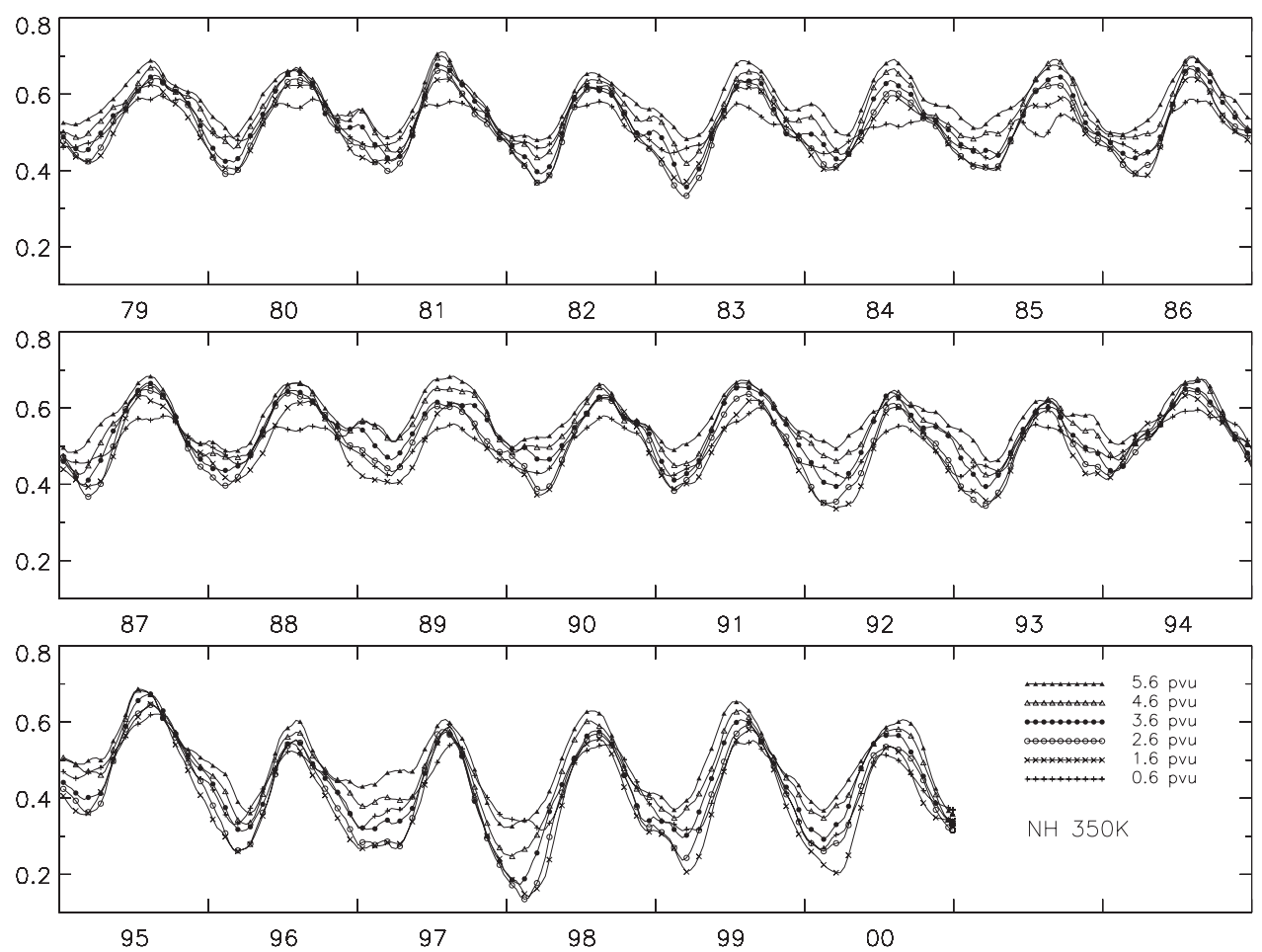

Figure 1. Exponential stretching rates of material contours initialized with $P V$ values of $0.6,1.6,2.6$, 3.6, 4.6, and 5.6 PVU (see key) on the $350 \mathrm{~K}$ isentropic surface calculated from contour advection simulations during the period 1979-2000. Values have been averaged in time with a running mean of 2 months.

and weak mixing by comparing the stretching rates of different contours.

\subsection{Effective Diffusivity and Equivalent Length}

[11] Another diagnostic for measuring the mixing properties of a flow is the effective diffusivity and the closely related equivalent length, as described by Nakamura [1996]. This diagnostic has been applied to atmospheric problems by Nakamura and Ma [1997], HS00a, HS00b, and AN01. It is calculated by solving the advection diffusion equation for a passive tracer field, using the analyzed winds on a given isentropic surface, and relaxing the tracer field back to an initialization profile (see HS00a and Shuckburgh et al. [2001] for further details). The advection is carried out here using SLIMCAT, an off-line chemical transport model which has been extensively used in simulations of stratospheric chemistry [Chipperfield, 1999].

[12] The effective diffusivity, $\kappa_{\text {eff, }}$ and equivalent length, $L_{\mathrm{eq}}$, are defined with respect to the advected tracer field, whose concentration is greater than a given value in a region bounded by a closed contour of length $L$. The equivalent length is related to this contour length by the inequality $L_{\mathrm{eq}} \leq L$, with equality when the gradient of the tracer field is uniform (in magnitude) around the contour (HS00a). Thus for a given area, larger values of $\kappa_{\text {eff }}$ and $L_{\text {eq }}$ imply a large enclosing contour and hence complicated geometry of the interface between the high and low tracer concentrations. In the work of Nakamura and Ma [1997] the effective diffusivity was calculated directly from an observed tracer (nitrous oxide) to examine mixing in the polar vortex.

\subsection{Contour Length and Equivalent Length}

[13] Equivalent length can also be calculated by taking advantage of the fine scales generated by contour advection calculations over a relatively short period, which provide a high-resolution tracer field. We use contour advection with surgery [Dritschel, 1989; Norton, 1994; Waugh and Plumb, 1994] to generate PV (our tracer) fields that contain smaller scales than do the raw analyses. The surgery is included to limit the small scales to $20 \mathrm{~km}$ for the sake of efficiency, the physical interpretation of which is the removal by $3 \mathrm{D}$ turbulent motions of vertical scales below, say, $100 \mathrm{~m}$, assuming a Prandtl number of 200. We initialize between 20 and 24 contours with PV values from \pm 0.1 PVU to $\pm 7 \mathrm{PVU}$ on the $330 \mathrm{~K}$ isentropic surface, from $\pm 0.1 \mathrm{PVU}$ to $\pm 8 \mathrm{PVU}$ on the $350 \mathrm{~K}$ surface, and from $\pm 0.4 \mathrm{PVU}$ to $\pm 12 \mathrm{PVU}$ on the $370 \mathrm{~K}$ surface. The large number of contours is used to obtain reasonable latitudinal resolution and coverage. These contours are then advected for 4 days, this time being long enough to generate fine-scale structure from the low-resolution reanalyses and short enough that the resulting PV fields remain realistic (see Scott et al. [2001] for a comparison of contour advection of PV with aircraft measurements of ozone in the subtropics). In the same manner as for the calculation of stretching rates, we use three simulations per month.

[14] The result of each simulation is gridded and the equivalent length is calculated according to the formula 

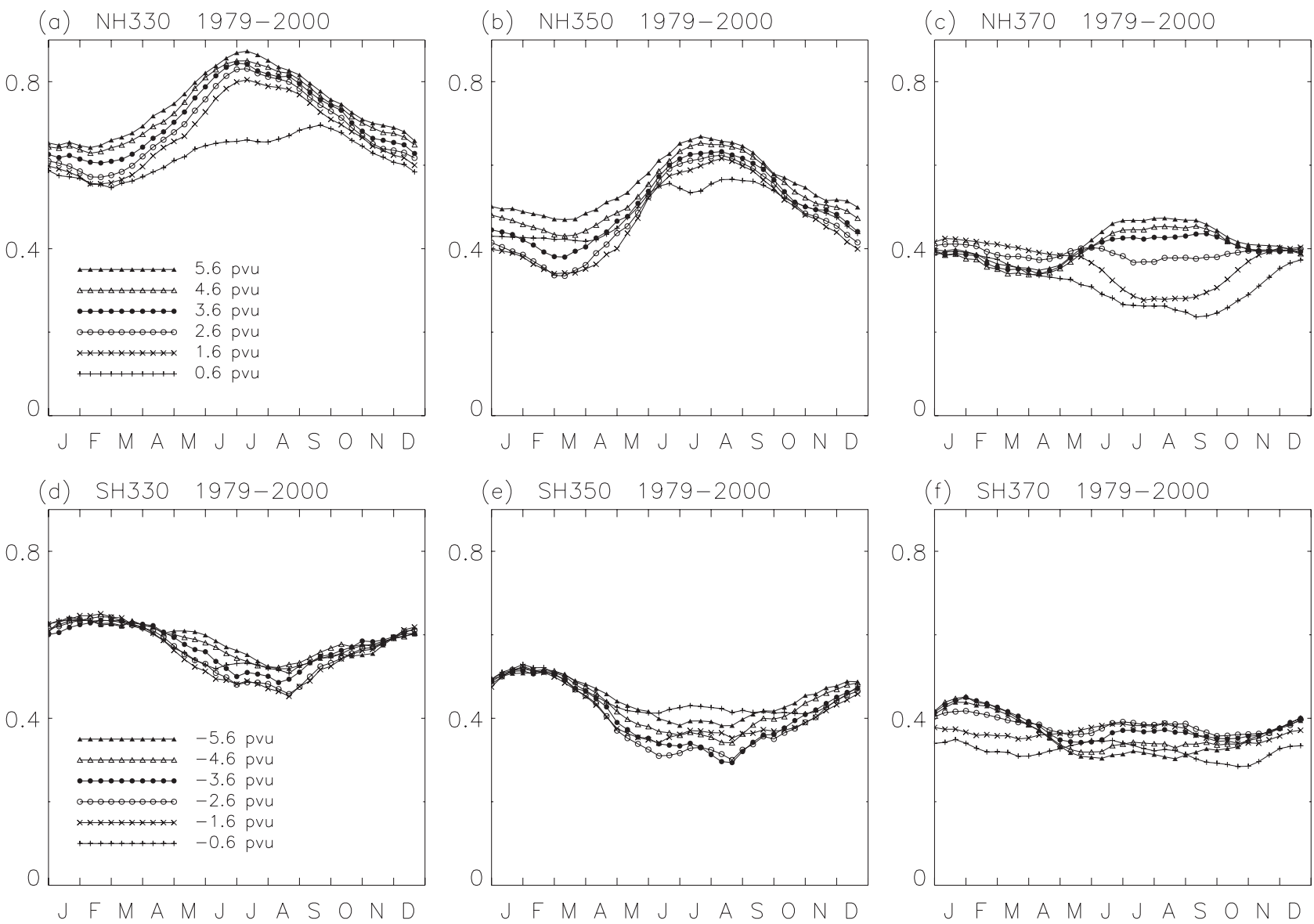

Figure 2. Seasonal climatology of stretching rates calculated during the 21 year period 1979-2000 for contours initialized with PV values of $\pm 0.6,1.6,2.6,3.6,4.6$, and 5.6 PVU in the Northern Hemisphere $(\mathrm{NH})$ (top panels) and Southern Hemisphere (SH) (bottom panels) on the (a and d) $330 \mathrm{~K}$, (b and e) 350 $\mathrm{K}$, and (c and $\mathrm{f}) 370 \mathrm{~K}$ isentropic surfaces.

given by Nakamura [1996], giving the equivalent length as a function of equivalent latitude and time. This quantity is then normalized by the length of the latitude circle at each equivalent latitude (so that the result is proportional to the effective diffusivity).

\section{Stretching Rates}

[15] As described in section 2.2, stretching rates are large when mixing is strong and are small when mixing is weak [see, e.g., Pierce and Fairlie, 1993; Chen, 1994; Ngan and Shepherd, 1999]. Stretching rates are also related to the Liapunov exponents of the flow [e.g., Ottino, 1989] and are essentially the rate at which fine scales are produced, on which 3D turbulent processes or molecular diffusivity may irreversibly mix fluid parcels. In this section we look at the distribution and variability of contour stretching rates from the period 1979-2000. In section 6 below we consider differences in the results obtained with the reanalyses (1979-1993) and operational analyses (1994-2000).

[16] Figure 1 shows the stretching rates of six contours initialized with the analyzed PV on the $350 \mathrm{~K}$ isentropic surface in the NH as a function of time from 1979-2000. Stretching rates were obtained for each simulation (three per month) and averaged in time with a running mean of
2 months. There is a clear seasonal cycle present in the stretching rates of each contour, indicating stronger mixing during the summer and weaker mixing during the winter. This pattern is consistent with the climatology of Rossby wave breaking derived by Postel and Hitchman [1999] using a PV gradient reversal criterion, which showed increased wave breaking during the summer (in each hemisphere) and related this to outflow from the main monsoon areas.

[17] A clearer picture of the seasonal cycle can be obtained by forming a climatology of stretching rates, shown in Figure 2, for years 1979-2000 (see section 6 below for a discussion of the differences between the analyses and reanalyses periods). This shows that the seasonal cycle has roughly the same structure over a fairly deep vertical range, which typically spans the vertical extent of the subtropical jet, and that the structure is also similar between hemispheres. Higher up, on $370 \mathrm{~K}$, the same seasonal cycle is still apparent on those contours with absolute PV values >2.6 PVU. The 0.6 and 1.6 PVU contours on $370 \mathrm{~K}$ have different time dependencies and indicate weak mixing in the tropics.

[18] If the tropopause is considered as a barrier to transport, then a natural definition of the location of the tropopause is the region where the mixing is weakest. In terms of stretching rates, the tropopause would thus be defined by the 

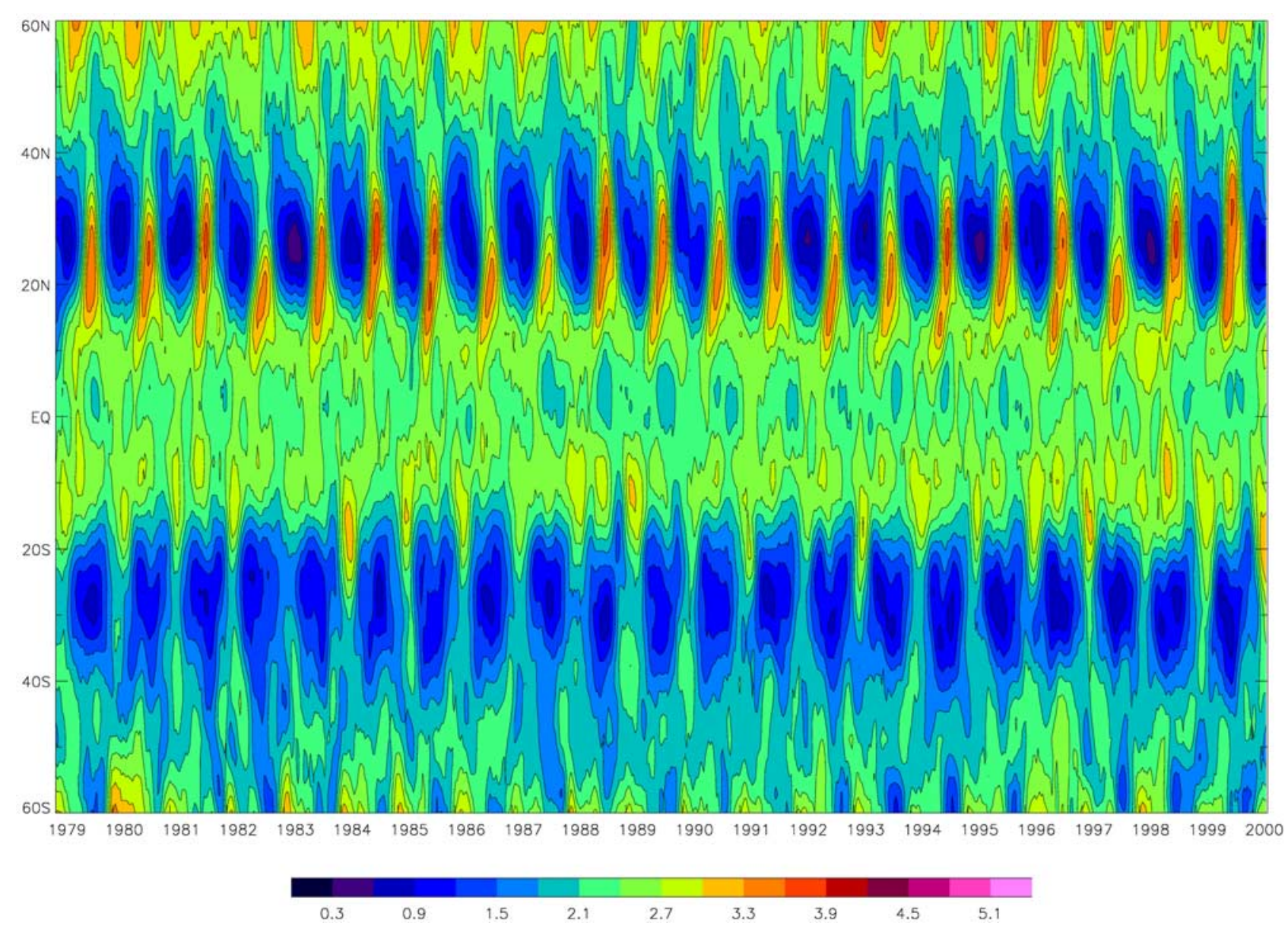

Figure 3. Equivalent length, $L_{\mathrm{eq}}$, on the $350 \mathrm{~K}$ isentrope calculated from the advection diffusion of a tracer field as a function of equivalent latitude and time during the period 1979-2000. The field has been averaged in time with a running mean of 1 month. The contour interval is 0.3 .

PV contour which exhibits the smallest stretching rates. Such a definition may be compared with traditional definitions as, for example, the location of the 2 PVU surface. This definition was tested by Bithell and Gray [1997], who considered stretching rates for a 6 day period in October 1990 using contours initialized with a range of PV values on various isentropic surfaces. Their results showed no single value of PV which yielded a minimum stretching rate and which could therefore be identified with the tropopause. On the other hand, as was pointed out in HSO0b, the period considered in that study (late summer) is one of strong mixing, and contours may experience stretching in a range of locations over the 6 day period, making it difficult to find a contour with a minimum stretching rate. It is possible that a clearer picture may be visible in winter, when the mixing is generally weaker.

[19] The above arguments are supported by the climatology shown in Figure 2. On the $350 \mathrm{~K}$ isentropic surface we see that during winter, when the mixing is generally weaker, there is a greater dependence of the stretching rates on the value of $\mathrm{PV}$ associated with the contour. Stretching rates are smallest for the 1.6 and 2.6 PVU contours in the NH and for the 2.6 and 3.6 PVU contours in the Southern Hemisphere (SH). On the $330 \mathrm{~K}$ isentrope during winter, somewhat lower values are found, although here the stretching rates show slightly less dependence on the PV values. Note that the dependence of stretching rates on the PV is considerably greater at all levels if the climatology is restricted to the more recent analysis period, 1995-2000; see the discussion in section 6.2 .

[20] On the $370 \mathrm{~K}$ isentrope during winter, stretching rates are minimum for generally higher values of PV, 3.64.6 PVU in the $\mathrm{NH}$ and 4.6-5.6 PVU in the SH, suggesting that the traditional $\mathrm{PV}$ definition of the tropopause (e.g., 2 PVU) may be less relevant here. However, this isentropic surface is located toward the very top of the troposphere and is intersected by the tropopause in low latitudes, so these results need to be interpreted with care. For example, during the summer the minimum stretching rates are found for the $0.6 \mathrm{PVU}$ contour, located very close to the equator. Such a minimum is possibly related to an equatorial barrier, also seen in the effective diffusivity calculations of HSOOb and AN01 and in section 4 below, and is likely not associated with any barrier effect of the tropopause in itself.

\section{Equivalent Length}

[21] The equivalent length (or effective diffusivity) has the advantage over the contour stretching rates described in section 2.2 of being a hybrid Eulerian-Lagrangian quantity that therefore avoids the problems, discussed in HSO0b, that 
might be encountered by purely Lagrangian techniques near weak barrier regions. An additional advantage is that the equivalent length is directly related to cross-contour fluxes [Nakamura, 1996]. Finally, it also provides a global picture of the mixing intensity, not restricted to a single PV contour.

[22] Figure 3 shows the logarithm of the equivalent length, $L_{\mathrm{eq}}$, for the period 1979-2000. As with the stretching rates, there is a clear seasonal cycle, with the lowest values of $L_{\text {eq }}$ (weakest mixing) occurring during winter. The weakest mixing occurs around $30^{\circ}$, centered on the winter subtropical jets. Such a correlation between low values of $L_{\text {eq }}$ and strong jets was pointed out by HS00b and AN01. In summer the weakest mixing appears at higher latitudes, consistent with the higher-latitude summer jets, while the summer minima near $60^{\circ} \mathrm{S}$ are associated with the lowermost part of the Antarctic polar vortex.

[23] To examine the seasonal cycle in more detail, we construct a climatology (years 1979-2000) of $L_{\text {eq }}$ on the $330 \mathrm{~K}, 350 \mathrm{~K}$, and $370 \mathrm{~K}$ isentropic surfaces (Figure 4). This shows more clearly the latitudinal migration of the minimum $L_{\text {eq }}$, which is visible in the NH on all levels. The climatology shows a strong summer increase in $L_{\text {eq }}$ near $30^{\circ}$ in the $\mathrm{NH}$, which is most pronounced at $350 \mathrm{~K}$ and $370 \mathrm{~K}$ and which is possibly related to the summer monsoon over Asia [Dunkerton, 1995; Dethof et al., 1999]. Note that there is also an increase in the value of the minimum $L_{\mathrm{eq}}$ at this time, that is, a general weakening of the subtropical tropopause barrier in summer. The latitudinal migration of the weak mixing region in the $\mathrm{SH}$ is less clear. On $330 \mathrm{~K}$ and $370 \mathrm{~K}$, there is an indication of a second minimum in $L_{\mathrm{eq}}$ near the equator, which indicates a weak equatorial barrier in the upper troposphere and helps to explain the lower stretching rates of the 0.6 PVU contour on these levels found in section 3 above.

[24] In the vertical, Figure 4 indicates that the weakest mixing is near $350 \mathrm{~K}$. This can be compared to the contour stretching rates discussed in section 3 , which were lowest at $350 \mathrm{~K}$ and $370 \mathrm{~K}$. Although the two diagnostics are in good agreement on each separate isentropic surface, these small differences in the vertical structure suggest the need for a deeper understanding of each diagnostic in typical velocity fields.

[25] Finally, from Figure $4 \mathrm{a}$ we see that there is a second region of weak mixing on $370 \mathrm{~K}$ near $60^{\circ}$ in the $\mathrm{SH}$ winter and spring. This is associated with the strong Antarctic polar vortex and is seen to persist late into spring, some time after the breakdown of the vortex at higher levels [Waugh et al., 1999]. This feature extends all the way down to $350 \mathrm{~K}$, as can be seen by the minima in Figures 3 and $4 \mathrm{~b}$.

\section{Equivalent Length From Contour Advection With Surgery}

[26] As discussed in section 2, the equivalent length, $L_{\text {eq }}$, can also be calculated from the high-resolution PV field resulting from contour advection of the analyzed PV field. Here contour advection provides additional information about the very small scales that are unresolved in the initial analyzed PV. The surgery provides a cutoff scale that prevents unlimited exponential lengthening of contours and hence permits the advection of many more contours than was possible when calculating contour stretching rates. This enables a latitudinal coverage to be obtained similar to (a) $370 \mathrm{~K}$

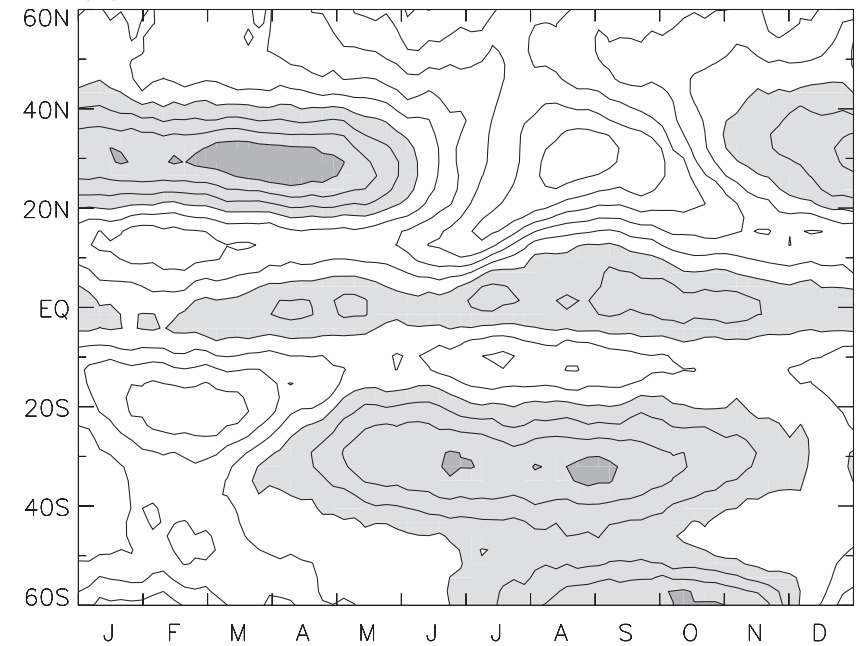

(b) $350 \mathrm{~K}$

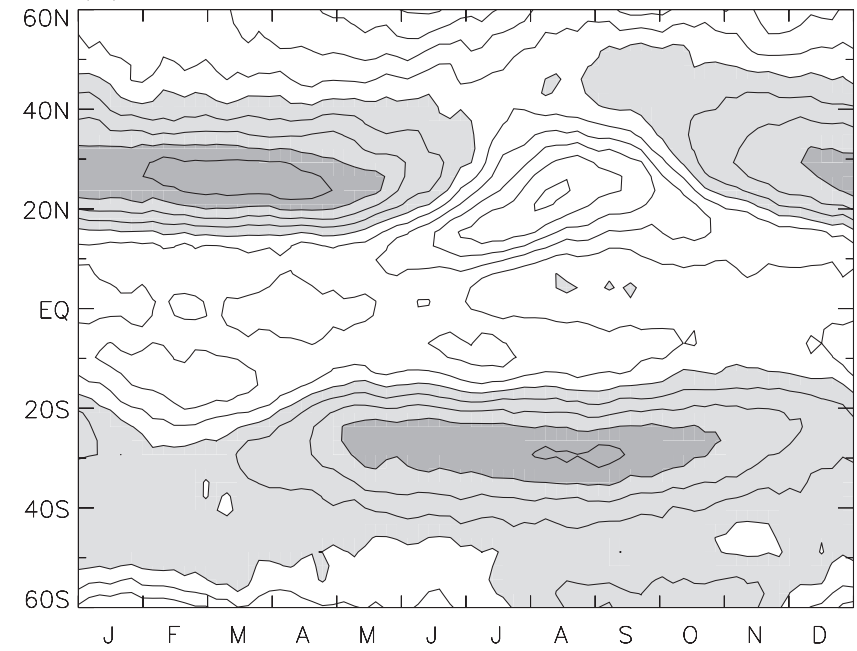

(c) $330 \mathrm{~K}$

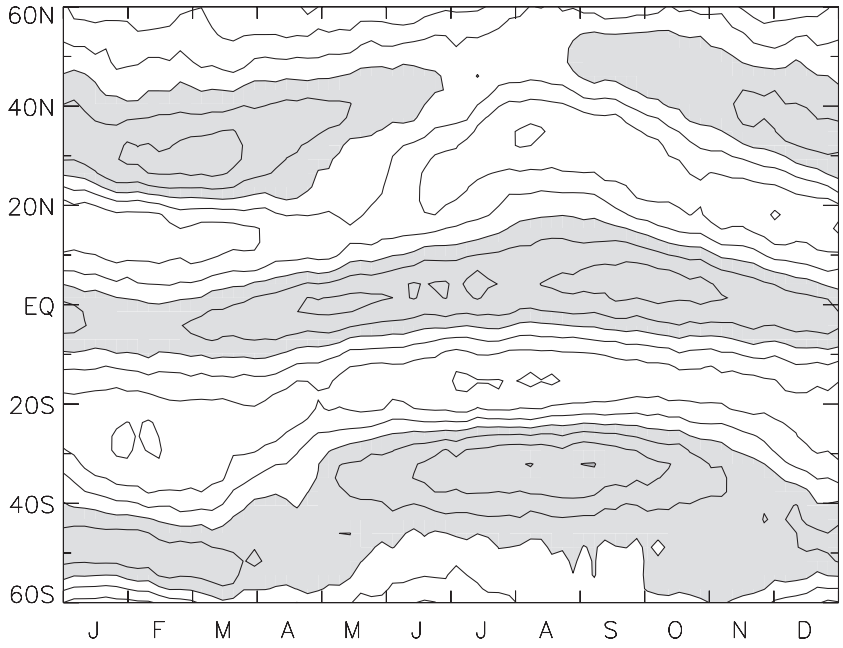

Figure 4. A climatology of equivalent length (calculated from the advection diffusion of a tracer field) for the period 1979-2000 as a function of equivalent latitude and time on (a) $370 \mathrm{~K}$, (b) $350 \mathrm{~K}$, and (c) $330 \mathrm{~K}$. Contours are as in Figure 3. 

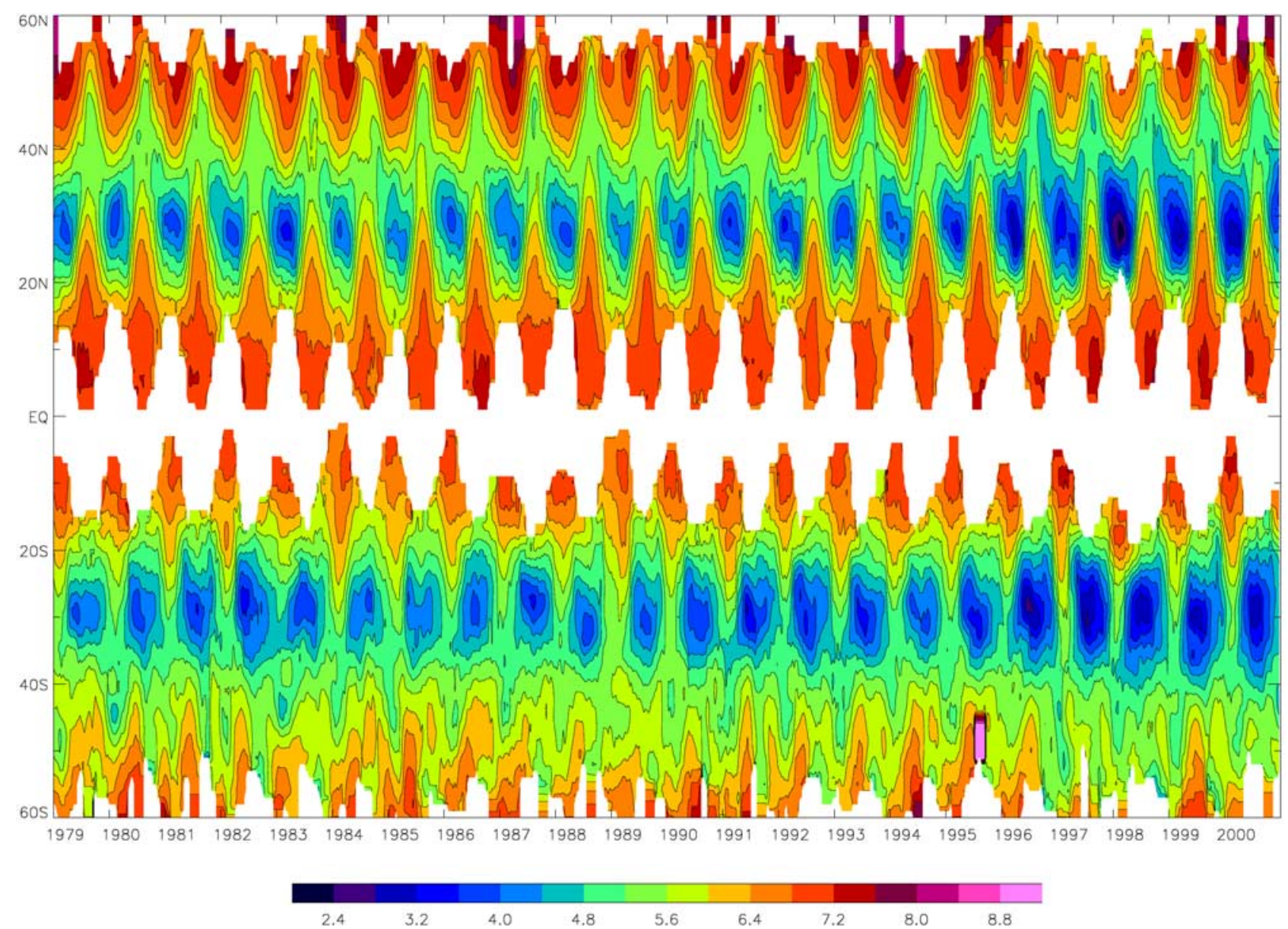

Figure 5. As in Figure 3, but for equivalent length calculated from contour advection with surgery of $\mathrm{PV}$ on the $350 \mathrm{~K}$ isentrope. The contour interval is 0.4 .

that of $L_{\text {eq }}$, derived from the advection of the tracer field discussed in section 4. Using PV contours between 0.1 and 8 PVU allows the calculation of $L_{\mathrm{eq}}$ between $\sim 20^{\circ}$ and $60^{\circ}$. At low levels in the tropics this method is unsuitable because of the weak dependence of PV on latitude.

[27] Figure 5 shows the equivalent length calculated from contour advection of the PV field on the $350 \mathrm{~K}$ isentropic surface for the years 1979-2000. There is broad agreement in the structure of the mixing intensity between this and the same diagnostic calculated from the tracer advection (Figure 3); in particular, there is good agreement of the minimum in mixing intensity and its latitude throughout the year. Both diagnostics also suggest similar differences between the $\mathrm{NH}$ and $\mathrm{SH}$ mixing characteristics. It should be emphasized that the methods of calculation are quite different, the first involving the advection diffusion equation and a passive tracer field and the second using the PV field from the analyses. One notable difference between the two diagnostics is the shift toward lower mixing values that can be seen in Figure 5 but not in Figure 3. This feature, which appears to arise from changes in the analyses, is discussed in section 6.2.

\section{Discussion}

\subsection{Seasonal Cycle}

[28] As already mentioned, one way to define the tropopause is by the region of weakest mixing. The stretching rates discussed in section 3 indicated the location of this region in terms of PV contours. However, the equivalent length, having a fuller latitudinal resolution, gives a more precise indication of the location of the tropopause region, which is easily expressed in terms of an equivalent latitude. Such a definition of the tropopause was used by HS00b on a 1.5 year period (HS00b, their Figure 5). The longer time period considered here allows us to see to what extent the features shown in that study are persistent features of the annual cycle.

[29] Figure 6 shows the value of PV and the associated equivalent latitude at the tropopause, that is, at the location of smallest $L_{\mathrm{eq}}$ at a given time, for the climatology of years 1979-2000. Both of the methods of calculation presented in sections 2.3 and 2.4 are used: contour advection with surgery (left-hand column) and tracer advection-diffusion (right-hand column). The equivalent latitude (dashed lines) shows the migration of the tropopause with time, as described in section 4 . The value of the PV at the tropopause also shows a similar variation with time, particularly on the $350 \mathrm{~K}$ and $370 \mathrm{~K}$ surfaces. (Note that the region of strong PV gradients associated with the subtropical jet also shows a similar seasonal migration as the minimum $L_{\mathrm{eq}}$. Thus the time variation of the $\mathrm{PV}$ at the minimum is due to the comparatively small shift of this minimum relative to that of the jet.) At $350 \mathrm{~K}$ in the $\mathrm{NH}$ the form of the time dependence is that of a rather abrupt change, from PV values of $\sim 2 \mathrm{PVU}$ for most of the year to $4-5 \mathrm{PVU}$ for a 

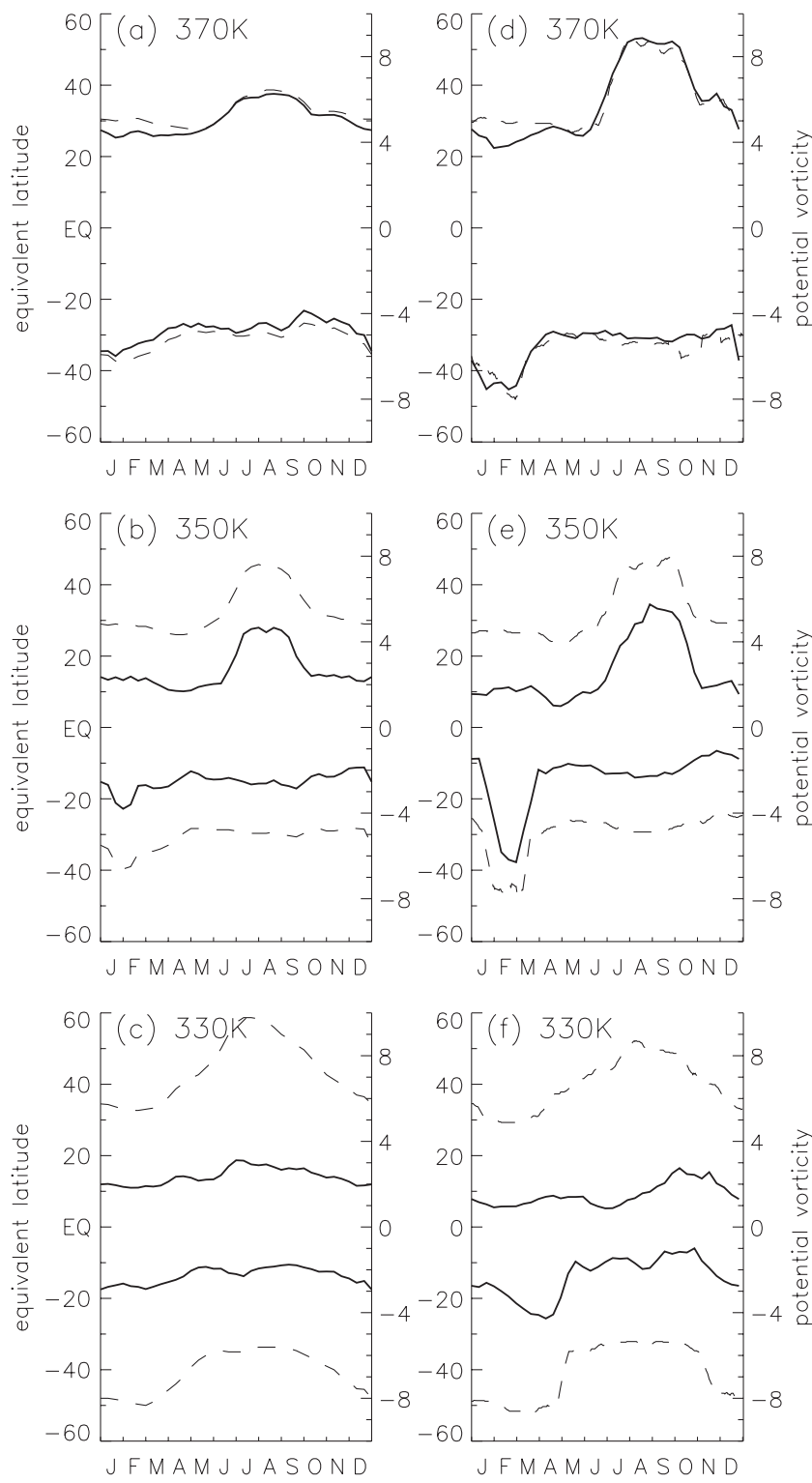

Figure 6. Equivalent latitude (dashed lines) of the local minimum of equivalent length calculated from tracer advection (left panels) and contour advection (right panels) based on a climatology of years 1979-2000 on (a and d) $370 \mathrm{~K}$, (b and e) $350 \mathrm{~K}$, and (c and f) $330 \mathrm{~K}$. The value of PV at this location is shown in solid.

short period of 2-3 months. The coincidence of the timing and duration of this maximum with the timing and duration of the summer monsoon supports the suggestion put forward in HS00b that the monsoon dynamics are responsible for the shift to higher PV values. A similar shift can also be seen at $350 \mathrm{~K}$ in the $\mathrm{SH}$, again possibly due to monsoon activity, although the two diagnostics show larger differences in the magnitude of this shift.

[30] The variations in PV at the tropopause on the other isentropic surfaces also show a tendency toward higher (absolute) PV values during the summer, although, again, the two diagnostics differ with regard to the magnitude of the shift, most notably at $370 \mathrm{~K}$. Finally, in both diagnostics, there is also a strong increase in PV at the tropopause with increasing potential temperature, from $\sim 2-3$ PVU on $330 \mathrm{~K}$ to $\sim 4-6$ PVU on $370 \mathrm{~K}$. This is consistent with the stretching rates discussed in section 3 , whose lowest values occurred on higher PV contours with increasing height.

[31] Figure 7 shows the values of the equivalent length at the tropopause, as defined above, together with the stretching rate of the 2.6 PVU contour for both hemispheres at $350 \mathrm{~K}$. There is broad agreement across all diagnostics, showing lower mixing during winter. Note that a comparison of the different diagnostics can only be made up to a constant scaling factor since in each case the magnitudes of the mixing depend upon such factors as data resolution, contour surgery scale, and diffusion coefficient. However, the variability of the mixing found in each diagnostic is comparable. Note that both equivalent-length diagnostics show a more abrupt change to higher summer values as well as a greater overall range of variability than do the stretching rates. Despite these differences, however, all diagnostics indicate stronger summertime mixing in the $\mathrm{NH}$ than in the $\mathrm{SH}$, consistent with the hypothesis that enhanced mixing is related to monsoon activity in each hemisphere.

\subsection{Interannual Variability}

[32] The diagnostics presented above show similar seasonal cycles and spatial distributions of the mixing intensity in the tropopause region. From Figures 1, 3, and 5, there is also a large amount of interannual variability in the mixing intensity. We now consider briefly some aspects of this variability and indicate those that appear to be a consequence of changes in the analyses and reanalyses.

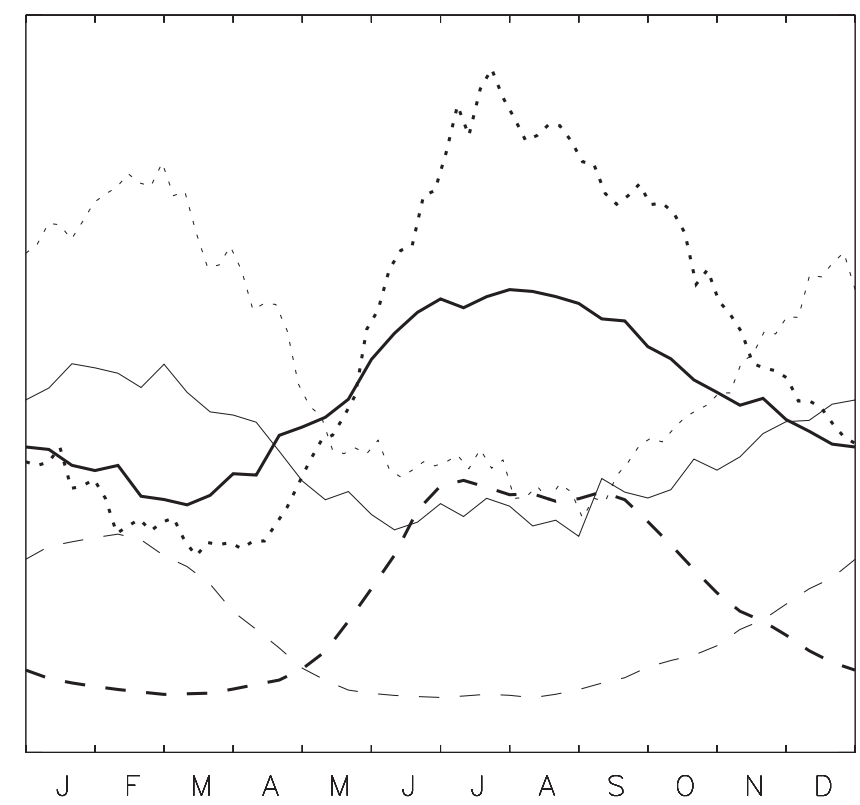

Figure 7. Seasonal climatology of stretching rates and equivalent length for the period $1979-2000$ at $350 \mathrm{~K}$. Shown are the stretching rate of the \pm 2.6 PVU (solid lines), the equivalent length at the tropopause (minimum of $L_{\mathrm{eq}}$ ) based on tracer advection (dotted lines), and the equivalent length at the tropopause based on contour advection (dashed lines). Bold lines denote $\mathrm{NH}$ values; thin lines denote $\mathrm{SH}$ values. 

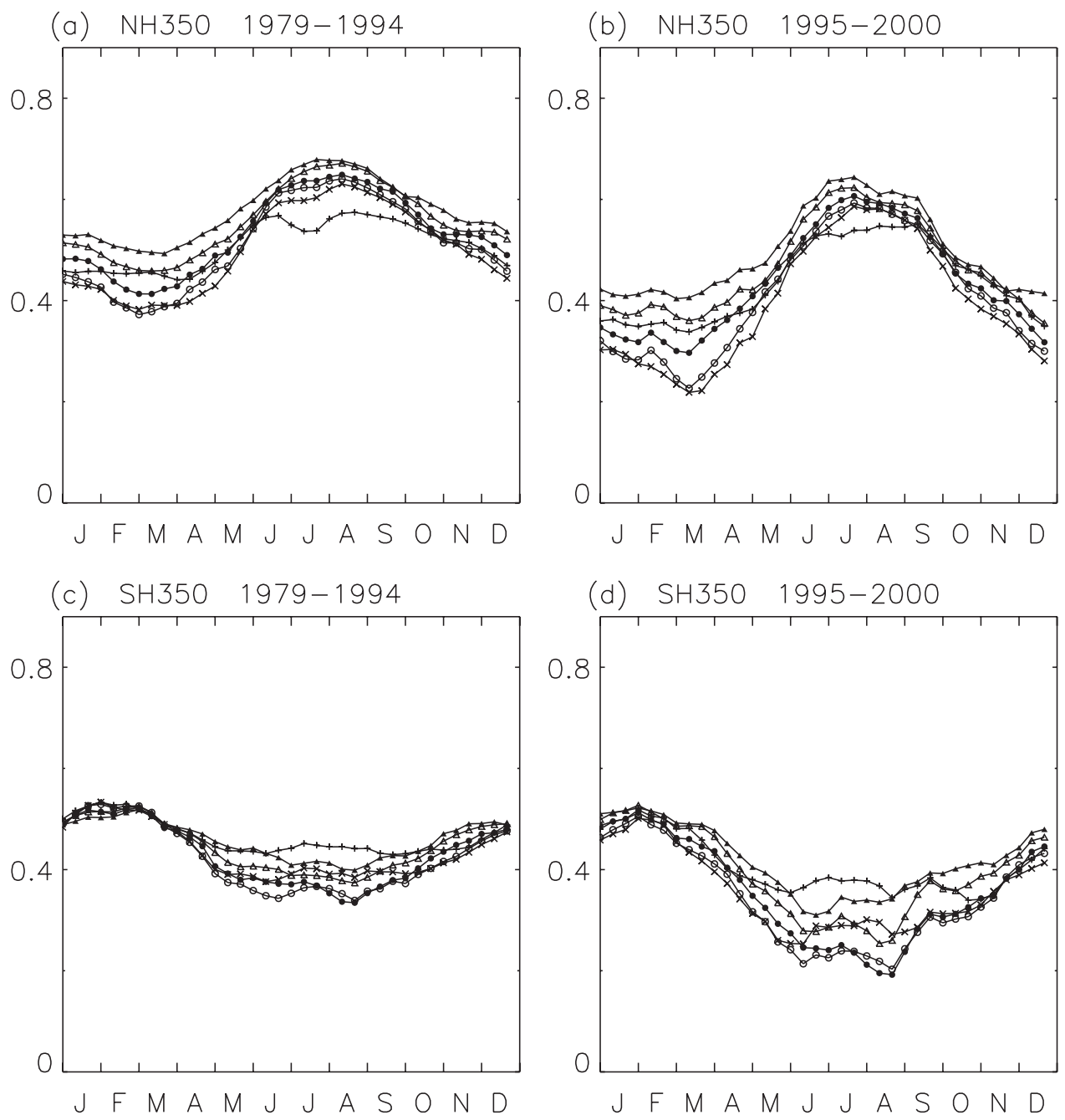

Figure 8. As in Figures $2 \mathrm{~b}$ and $2 \mathrm{e}$, but calculated using ( $\mathrm{a}$ and $\mathrm{c}$ ) the ERA-15 reanalyses for the 16 year period 1979-1993 and using (b and d) the operational analyses for the 6 year period 1994-2000.

[33] The full time series of stretching rates shown in Figure 1 indicates an abrupt change to generally weaker mixing intensities around 1995-1996. Because the change is rather abrupt, it appears likely that it results from differences between the two data sets (arising from different representations of physical processes, model resolution, and assimilation schemes) rather than from any atmospheric trend. In fact, the timing of the change coincides with the introduction of the $3 \mathrm{D}$ variational assimilation in the ECMWF analyses cycle in January 1996 [Courtier et al., 1998]. This decrease in mixing was also found by Morgenstern and Carver [2001] using a filament detection algorithm and contour advection based on the same analyses.

[34] Dividing the climatology of stretching rates shown in Figure 2 into two parts, based on the ERA-15 reanalyses (1979-1993) and on the operational analyses (1994-2000), Figure 8 indicates aspects of this change. First, on $350 \mathrm{~K}$ as well as on the other isentropic surfaces (not shown) the seasonal cycle in the analyses is considerably stronger than that in the reanalyses; that is, the difference between summer and winter mixing intensities is greater in the more recent data set. Second, the dependence of stretching rate on
PV contour during the winter is also greater in the analyses (again, this feature is seen on all isentropic surfaces considered). Thus, to the extent that the tropopause can be considered a mixing barrier and have low stretching rates relative to other regions, the more recent analyses provide a clearer identification of the tropopause.

[35] The equivalent length calculated by contour advection of PV (Figure 5) suggests a similar shift to weaker mixing intensity at the tropopause, while the same quantity calculated from the advection diffusion equation (Figure 3) does not. Figure 9 summarizes the variation at the tropopause of all diagnostics for the period 1979-2000, showing the stretching rates of the \pm 2.6 PVU contour and the value of the local minimum of $L_{\text {eq }}$, calculated using both methods described in sections 2.3 and 2.4. As stated in this section, shifts to weaker mixing with increasing time are probably associated with changes in the analyzed data set.

[36] One possible reason why the stretching rates and the contour advection-based $L_{\mathrm{eq}}$ show such a shift while the tracer advection-based $L_{\mathrm{eq}}$ does not is that the former two quantities are calculated using the PV field and the wind field, whereas the latter is calculated using the wind field alone. Since the PV field is a derived quantity, it often 

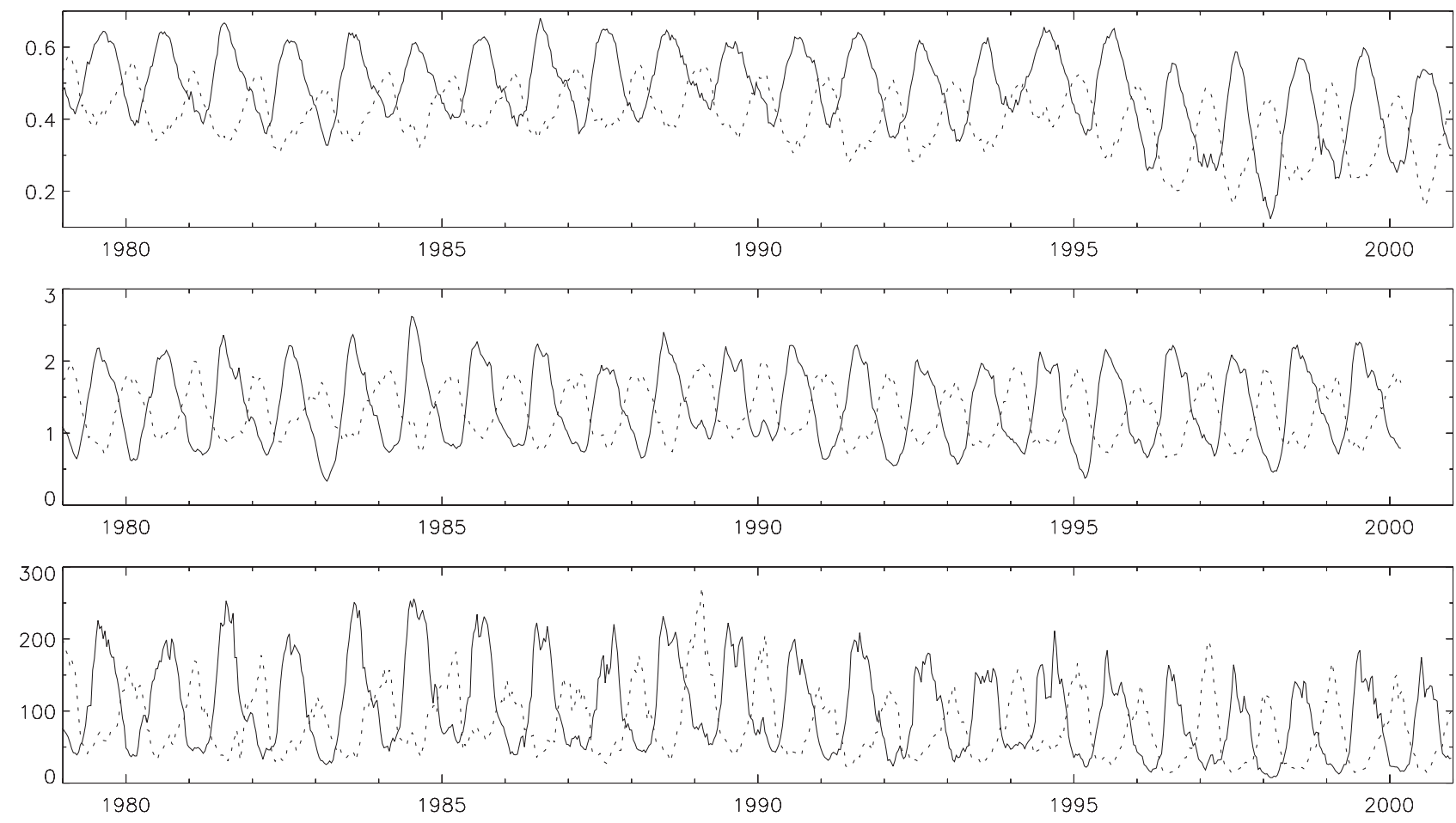

Figure 9. (a) Stretching rates of the 2.6 PVU contour, and the equivalent length at the tropopause (minimum of $L_{\mathrm{eq}}$ ) calculated from (b) tracer advection and from (c) contour advection for the period 1979-2000. Solid lines denote NH values; dotted lines denote SH values.

exhibits a large amount of noise, more particularly in the reanalyses than in the later analyses. When PV features associated with this noise are advected by the wind field, large stretching rates and fine-scale filamentary structures will be produced, indicating stronger mixing in any $\mathrm{PV} /$ advective-based diagnostic.

[37] Ignoring the later shift to weaker mixing, all three diagnostics indicate a certain correspondence with El NiñoSouthern Oscillation (ENSO) phase in both hemispheres. For example, the strong El Niño years of 1983 and 1998 show up as local minima in the wintertime values of the stretching rates and both equivalent length diagnostics. In contrast, during the strong La Niña year of 1989, wintertime mixing intensities are markedly higher than in other years. The relationship between ENSO phase and the variation in the subtropical jet and wave propagation characteristics near the tropopause has been the subject of several recent studies. Matthews and Kiladis [1999] and Waugh and Polvani [2000] showed a decrease in equatorward wave propagation in El Niño years on account of a stronger and more zonal subtropical jet over the Pacific Ocean. Shapiro et al. [2001] found Pacific conditions during El Niño more favorable to LC1-type [Thorncroft et al., 1993] baroclinic development and conditions during La Niña more favorable to LC2-type development. Scott and Cammas [2002] found consistently weaker wave breaking over the Pacific during El Niño years. Although the changes in the analyses and reanalyses make it difficult to relate any variability in the current data set to variability in the real atmosphere, the weaker mixing in 1983/1998 and stronger mixing in 1989 are suggestive of an influence of the ENSO phase and are consistent with earlier studies.
[38] Interannual variability in mixing is also visible at other latitudes. For example, at higher latitudes on $370 \mathrm{~K}$ in the $\mathrm{SH}$ the distinct local minimum in $\kappa_{\text {eff }}$ in late spring (see Figure 4c) is probably due to the persistence of the lowermost part of the winter stratospheric polar vortex, or subvortex. Consideration of the full time series of $L_{\mathrm{eq}}$ on this level (not shown) indicates a recent trend toward weaker mixing in both hemispheres, which is possibly related to the recent cooling trend of the polar vortex, although, again, changes in the analyses data set cannot be ruled out.

\section{Conclusions}

[39] We have presented two diagnostics of mixing activity: contour stretching rates and effective diffusivity/equivalent length. The former is a purely Lagrangian diagnostic based on the advection of material contours using meteorological winds and potential vorticity on isentropic surfaces in the upper troposphere/lower stratosphere region. The latter diagnostic, a hybrid Eulerian-Lagrangian diagnostic, has been calculated in two ways, the first involving the advection diffusion of a tracer field by meteorological winds and the second involving the generation of a high-resolution PV field by contour advection with surgery. The mixing diagnostics are calculated over the period 1979-2000, enabling a consideration of both seasonal and interannual variability of the mixing activity near the tropopause as well as providing an indication of variability induced by the ongoing development of the model-data system used to construct the meteorological analyses. The latter suggests that changes in the model assimilation cycle of the ECMWF 
analyses affect the mixing properties of the tropopause region by accentuating the mixing barrier in the more recent analyses. Both diagnostics revealed generally stronger mixing in the summer and weaker mixing in the winter as well as stronger mixing at $330 \mathrm{~K}$ than higher up, in agreement with previous studies.

[40] Contour stretching rates based on standard contour advection (i.e., without surgery) of PV contours showed that stretching rates in the winter, when mixing is generally weaker, can depend substantially (especially in the 19952000 analyses) on the PV value of the contour. The dependence on PV during winter suggests that stretching rates can give some indication about the location of the tropopause if it is interpreted as the region of weakest mixing, that is, if the tropopause is treated as a transport barrier. The wintertime stretching rates in this study are weakest at $\sim 2$ PVU $(\mathrm{NH})$ and -2 to -3 PVU (SH) at $330 \mathrm{~K}$ and $350 \mathrm{~K}$ and at $4 \mathrm{PVU}(\mathrm{NH})$ and $-5 \mathrm{PVU}(\mathrm{SH})$ at $370 \mathrm{~K}$.

[41] The equivalent length, $L_{\mathrm{eq}}$, calculated both from advection-diffusion of a tracer field and from contour advection with surgery of $\mathrm{PV}$, revealed mixing intensities that were in broad agreement with the stretching rates. Again, there was considerable seasonal variability in the location of the tropopause (as defined by the local minimum of $L_{\text {eq }}$ ) and in the strength of the associated transport barrier as well as seasonal variability in the values of PV there. In particular, the PV values at the local minimum of $L_{\mathrm{eq}}$ on $350 \mathrm{~K}$ were larger during the summer monsoon seasons, in agreement with the findings of HS00b and AN01 and with the earlier studies of Dunkerton [1995] and Dethof et al. [1999]. The 21 year data set and the two separate methods used to calculate $L_{\mathrm{eq}}$ demonstrate the robustness of this feature.

[42] Finally, both diagnostics exhibited interannual variability consistent with the influence of ENSO on wave breaking at the subtropical jet, although the nonuniformity of the 21 year data set makes a direct correlation difficult. For example, all diagnostics showed weaker winter mixing during the strong El Niño years 1983 and 1998 and stronger mixing during the La Niña year 1989. Stronger and more zonal subtropical jets over the Pacific during the warm phase can lead to weaker wave breaking at the tropopause, as discussed in a number of recent studies [Matthews and Kiladis, 1999; Waugh and Polvani, 2000; Shapiro et al., 2001; Scott and Cammas, 2002]. Ongoing work is considering whether a reduction in monsoon intensity during the warm ENSO phase can similarly lead to weaker mixing over Asia during the NH summer.

[43] Acknowledgments. The authors wish to thank Martyn Chipperfield, Alan Iwi, and Warwick Norton for discussions and computational assistance. Computing resources were provided by the UK Universities Global Atmospheric Modelling Programme. RKS was supported by the European Commission (EC) under contract EVK2-1999-00015; EFS was supported by the EC under contract ENV4-CT97-0540 and by an EC Marie Curie Fellowship under contract HPMF-CT-2000-00636.

\section{References}

Allen, D. R., and N. Nakamura, A seasonal climatology of effective diffusivity in the stratosphere, J. Geophys. Res., 106, 7917-7935, 2001.

Appenzeller, C., H. C. Davies, and W. A. Norton, Fragmentation of stratospheric intrusions, J. Geophys. Res., 101, 1435-1456, 1996.
Baray, J.-L., V. Daniel, G. Ancellet, and B. Legras, Planetary-scale tropopause folds in the southern subtropics, Geophys. Res. Lett., 27, 353-356, 2000.

Bithell, M., and L. J. Gray, Contour lengthening rates near the tropopause, Geophys. Res. Lett., 24, 2721-2724, 1997.

Bowman, K. P., Large-scale isentropic mixing properties of the Antarctic polar vortex from analyzed winds, J. Geophys. Res., 98, 23,013-23,027, 1993.

Bowman, K. P., and Y. Hu, Tropical mixing barriers in the lower stratosphere in the Geophysical Fluid Dynamics Laboratory SKYHI model, J. Geophys. Res., 102, 21,465-21,478, 1997.

Chen, P., The parmeability of the Antarctic vortex edge, J. Geophys. Res., 99, 20,563-20,571, 1994

Chen, P., Isentropic cross-tropopause mass exchange in the extratropics, J. Geophys. Res., 100, 16,661-16,673, 1995

Chipperfield, M. P., Multiannual simulations with a three-dimensional chemical transport model, J. Geophys. Res., 104, 1781-1805, 1999.

Courtier, P., E. Andersson, W. Heckley, J. Pailleux, D. Vasiljevic, M. Hamrud, A. Hollingsworth, E. Rabier, and M. Fisher, The ECMWF implementation of three-dimensional variational assimilation (3D-Var). I: Formulation, $Q$. J. R. Meteorol. Soc., 124, 1783-1807, 1998.

Dethof, A., A. O'Neill, J. M. Slingo, and H. G. J. Smit, A mechanism for moistening the lower stratosphere involving the Asian summer monsoon, O. J. R. Meteorol. Soc., 125, 1079-1106, 1999.

Dritschel, D. G., Contour dynamics and contour surgery: Numerical algorithms for extended, high-resolution modelling of vortex dynamics in two-dimensional, inviscid, incompressible flows, Comput. Phys. Rep., 10, $78-146,1989$.

Dunkerton, T. J., Evidence of meridional motion in the summer lower stratosphere adjacent to monsoon regions, J. Geophys. Res., 100, 16,67516,688, 1995.

Gibson, J. K., P. Kallberg, S. Uppala, A. Hernandez, A. Nomura, and E. Serrano, ERA Description, ECMWF Re-analyses Proj. Rep. Ser, vol. 1, Eur. Cent. for Medium-Range Weather Forecasts, Reading, U. K., 1997.

Haynes, P., and E. Shuckburgh, Effective diffusivity as a diagnostic of atmospheric transport: 1. Stratosphere, J. Geophys. Res., 105, 22,77722,794, 2000a.

Haynes, P., and E. Shuckburgh, Effective diffusivity as a diagnostic of atmospheric transport: 2. Troposphere and lower stratosphere, J. Geophys. Res., 105, 22,795-22,810, 2000b.

Holton, J. R., P. H. Haynes, M. E. McIntyre, A. R. Douglass, R. B. Rood, and L. Pfister, Stratosphere-troposphere exchange, Rev. Geophys., 33, 403-439, 1995

Juckes, M. N., A shallow water model of the winter stratosphere, J. Atmos. Sci., 46, 2934-2955, 1989.

Klinker, E., F. Rabier, G. Kelly, and J. F. Mahfouf, The ECMWF operational implementation of four-dimensional variational assimilation. III: Experimental results and diagnostics with operational configuration, Q. J. R. Meteorol. Soc., 126, 1191-1215, 2000.

Mariotti, A., M. Moustaoui, B. Legras, and H. Teitelbaum, Comparison between vertical ozone soundings and reconstructed potential vorticity maps by contour advection with surgery, J. Geophys. Res., 102, 61316142, 1997.

Matthews, A. J., and G. N. Kiladis, Interactions between ENSO, transient circulation, and tropical convection over the Pacific, J. Clim., 12, 30623086, 1999.

Morgenstern, O., and G. D. Carver, Comparison of cross-tropopause transport and ozone in the upper troposphere and lower stratosphere region, J. Geophys. Res., 106, 10,205-10,221, 2001.

Nakamura, N., Two-dimensional mixing, edge formation, and permeability diagnosed in an area coordinate, J. Atmos. Sci., 53, 1524-1537, 1996.

Nakamura, N., and J. Ma, Modified Lagrangian-mean diagnostics of the stratospheric polar vortices: 2 . Nitrous oxide and seasonal barrier migration in the cryogenic limb array etalon spectrometer and SKYHI general circulation model, J. Geophys. Res., 102, 25,721-25,735, 1997.

Ngan, K., and T. G. Shepherd, A closer look at chaotic advection in the stratosphere. part II: Statistical diagnostics, J. Atmos. Sci., 56, 41534166, 1999.

Norton, W. A., Breaking Rossby waves in a model stratosphere diagnosed by a vortex-following coordinate system and a technique for advecting material contours, J. Atmos. Sci., 51, 654-673, 1994.

Ottino, J. M., The Kinematics of Mixing: Stretching, Chaos and Transport, Cambridge Univ. Press, New York, 1989.

Pierce, R. B., and T. D. A. Fairlie, Chaotic advection in the stratosphere: Implications for the dispersal of chemically perturbed air from the polar vortex, J. Geophys. Res., 98, 18,589-18,595, 1993.

Pierrehumbert, R. T., and H. Yang, Global chaotic mixing on isentropic surfaces, J. Atmos. Sci., 50, 2462-2480, 1993.

Polvani, L. M., D. W. Waugh, and R. A. Plumb, On the subtropical edge of the stratospheric surf zone, J. Atmos. Sci., 52, 1288-1309, 1995. 
Postel, G. A., and M. H. Hitchman, A climatology of Rossby waves breaking along the subtropical tropopause, J. Atmos. Sci., 56, 359-373, 1999

Scott, R. K., and J.-P. Cammas, Wave breaking and mixing at the subtropical tropopause, J. Atmos. Sci., 59, 2347-2361, 2002.

Scott, R. K., J.-P. Cammas, P. Mascart, and C. Stolle, Stratospheric filamentation into the upper tropical troposphere, J. Geophys. Res., 106, $11,835-11,848,2001$

Shapiro, M. A., H. Wernli, N. A. Bond, and R. Langland, The influence of the 1997-1999 ENSO on extratropical baroclinic life cycles over the eastern North Pacific, Q. J. R. Meteorol. Soc., 127, 331-342, 2001.

Shepherd, T. G., J. N. Koshnyk, and K. Ngan, On the nature of large-scale mixing in the stratosphere and mesosphere, J. Geophys. Res., 105, $12,433-12,446,2000$.

Shuckburgh, E., W. Norton, A. Iwi, and P. Haynes, Influence of the quasibiennial oscillation on isentropic transport and mixing in the tropics and subtropics, J. Geophys. Res., 106, 14,327-14,337, 2001

Thorncroft, C. D., B. J. Hoskins, and M. E. McIntyre, Two paradigms of baroclinic-wave life-cycle behaviour, Q. J. R. Meteorol. Soc., 119, 17$55,1993$.
Waugh, D. W., and R. A. Plumb, Contour advection with surgery: A technique for investigating finescale structure in tracer transport, J. Atmos. Sci., 51, 530-540, 1994.

Waugh, D. W., and L. M. Polvani, Climatology of intrusions into the tropical upper troposphere, Geophys. Res. Lett., 27, 3857-3860, 2000.

Waugh, D. W., W. J. Randel, S. Pawson, P. A. Newman, and E. R. Nash, Persistence of the lower stratospheric polar vortices, J. Geophys. Res., 104, 27,191-27,201, 1999.

J.-P. Cammas, Laboratoire d'Aérologie, 14, avenue E. Belin, F-31400 Toulouse, France. (camjp@aero.obs-mip.fr)

B. Legras and E. F. Shuckburgh, Laboratoire de Météorologie Dynamique, Ecole Normale Superieure, 24 rue Lhomond, F-75231 Paris cedex 05, France. (legras@ens.fr; efs@1md.ens.fr)

R. K. Scott, Department of Applied Physics and Applied Mathematics, Columbia University, 500 West 120th Street, New York, NY 10027, USA. (scott@appmath.columbia.edu) 